\title{
THE HULL OF HOLOMORPHY OF A NONISOTROPIC BALL IN A REAL HYPERSURFACE OF FINITE TYPE
}

\author{
A. BOGGESS, R. DWILEWICZ, AND A. NAGEL
}

\begin{abstract}
We show that the hull of holomorphy of a nonisotropic ball in a real hypersurface of finite type in $C^{n}$ contains an open set in $C^{n}$ which emanates from the hypersurface a distance which is proportional to the length of the minor axis of the nonisotropic ball. In addition, we prove a maximal function estimate for plurisubharmonic functions which is important in the study of boundary values of holomorphic functions.
\end{abstract}

\section{INTRODUCTION}

Hans Lewy [L] first introduced the problem of the local holomorphic extension of $\mathbf{C R}$ functions when he showed that near a point on a real hypersurface $M$ in $C^{n}$ of type 2, i.e., a point where the first Levi form is nonvanishing, CR functions locally extend as holomorphic functions to an open set in $C^{n}$. That is, the local hull of holomorphy of such a hypersurface contains an open set in $C^{n}$. Since then, there has been an abundance of literature on $\mathbf{C R}$ extension. In particular, Baouendi and Treves [BT] and later Boggess and Pitts [BP] generalized Lewy's result to points of finite but higher type, i.e., points where the first Levi form vanishes but where some higher order Levi form is nonvanishing. Our goal in this paper is to give a precise description of the size of the hull of holomorphy of an open subset $\omega$ of $M$ in terms of the size of $\omega$ and the geometry of $M$.

Some preliminary results of this type are implicit in the work of [L] and [BP]. If $p \in M$ is a point of type $m<\infty$, then it is shown in [L] (for $m=2$ ) and [BP] (for $2 \leq m<\infty$ ) that if $\omega$ contains the Euclidean ball of radius $\delta$ in $M$ centered at $p$, then the hull of $\omega$ contains an open set in $C^{n}$ which emanates from $M$ a distance of $C \delta^{m}$, where $C$ is some proportionality constant. However, the proportionality constant $C$ depends on various derivatives of the defining functions of $M$ which may get uncontrollably small, for example, at points of type 2 which are near points of higher type. Instead, it would be desirable to have a size estimate for the local hull of holomorphy which is uniform at all points $M$, regardless of type.

Received by the editors January 3, 1989.

1980 Mathematics Subject Classification (1985 Revision). Primary 32E20, 32F25; Secondary $32 \mathrm{~F} 30$. 
If $M$ is a hypersurface of finite type, there is an intrinsic nonisotropic metric on $M$ which reflects the possible degeneracy of the Levi form. This metric was defined and some of its properties stated in [NSW1], and the corresponding nonisotropic balls $B_{M}(p, \delta)$ have been used in other contexts (see [NSW2, N]). The nonisotropic balls $B_{M}(p, \delta)$ are smaller than the corresponding isotropic balls defined by the ambient Euclidean metric. In $[\mathrm{N}]$ this nonisotropic metric was used to study the local hull of holomorphy of nonisotropic balls for certain very special hypersurfaces $M$ of the form

$$
M=\left\{(z, w) \in C^{2} ; \operatorname{Im}(w)=P(z)\right\},
$$

where $P$ is a subharmonic, nonharmonic polynomial.

In this paper, we show that for a general hypersurface $M \subset C^{n}$ of finite type, there is a size estimate for the local hull of holomorphy of the associated nonisotropic balls $B_{M}(p, \delta)$ which is uniform at all points of $M$. These size estimates are defined in terms of quantity

$$
\Lambda_{M}(p, \delta)=\sum_{j=2}^{m_{0}} \lambda_{j}(p) \delta^{j},
$$

where $m_{0}$ is the maximum type of any point on $M$, and $\lambda_{j}(p)$ involves commutators of length $j$ of vector fields from the complexified holomorphic tangent bundle of $M$ and vanish when the Levi form at $p$ vanishes to order $j-2$. Thus at points of type $2, \Lambda_{M}(p, \delta) \approx \delta^{2}$ for small $\delta$ while at a point of maximum type, $\Lambda_{M}(p, \delta) \approx \delta^{m_{0}}$ for small $\delta$. For larger $\delta$, the quantity $\Lambda_{M}(p, \delta)$ provides a smooth transition between points of type 2 and points of higher type. This allows us to give a uniform description of the hull of holomorphy of the nonisotropic balls $B_{M}(p, \delta)$, which are essentially twisted ellipsoids centered at $p$ of radius $\delta$ in the holomorphic tangent space directions, and of radius $\Lambda_{M}(p, \delta)$ in the totally real direction.

If the hypersurface $M$ bounds a pseudoconvex domain $D$, then one expects that the hull of holomorphy will always be on the convex side of the surface $M$. On the other hand, if we only assume that $M$ is of finite type, the hull of holomorphy may be on either (or both) sides, as is shown in the work of Baouendi and Treves [BT2]. Thus our main theorem (Theorem 2.4) comes in two parts. The first part states (roughly) that if the surface $M$ is of finite type, then the hull of holomorphy of a nonisotropic ball $B_{M}(p, \delta)$ contains an open set in $C^{n}$ which lies on one (or both) sides of $M$, and which emanates a distance at least $\tau_{0} \Lambda_{M}(p, \delta)$ from $M$ in the same normal direction over a smaller ball $B_{M}\left(p, \varepsilon_{0} \delta\right)$, where $\tau_{0}$ and $\varepsilon_{0}$ are uniform constants independent of $p$ and $\delta$. The second part states (roughly) that if in addition $M$ bounds a pseudoconvex domain, then the hull of holomorphy will contain an open set described as follows: for each point $q \in B_{M}(p, \delta)$, the open set contains those points on the normal line to $M$ through $q$ which lie on the convex side of $M$, and whnse distance from $q$ is at most $\tau_{0} \Lambda_{M}\left(p, \delta_{q}\right)$. Here $\tau_{0}$ is again 
a uniform constant independent of $p, q$, and $\delta$, and $\delta_{q}$ is the radius of the largest nonisotropic ball centered at $q$ contained in $B_{M}(p, \delta)$. The main difference to notice in these two cases is that in the pseudoconvex case, one has control over the size and direction of the open set above every point of the ball $B_{M}(p, \delta)$, while if one only assumes finite type, one has this uniform directional control only over a smaller ball $B_{M}\left(p, \varepsilon_{0} \delta\right)$.

Our approach to the problem of estimating the hull of holomorphy uses the techniques of analytic discs. Thus we show (Theorem 2.5) that we can fill out the required open sets in $C^{n}$ with the centers of analytic discs whose boundaries lie to one side of the hypersurface $M$. We can also use our analytic disc result to show (Theorem 2.6) that if $M$ bounds a pseudoconvex domain $D$, then the value of a plurisubharmonic function $u$ at a point $z \in D$ can be estimated by an average of $|u|$ over an appropriately chosen nonisotropic ball on $M$. This estimate was obtained in very special cases in [N], and is of independent interest since the analogous result for subharmonic functions and Euclidean balls in $R^{n}$ is false.

In $C^{2}$, convex examples show that our results our sharp. That is, the hull of holomorphy of a nonisotropic ball in a real hypersurface in $C^{2}$ is up to proportionality constants no bigger than the set described in Theorem 2.4. However, for $n>2$, our results are not optimal because our ellipsoids do not distinguish between different directions within the holomorphic tangent space. For example, if

$$
M=\left\{\operatorname{Re} z_{1}=\left|z_{2}\right|^{2}+\left|z_{3}\right|^{4}\right\},
$$

then the hull of holomorphy of our ellipsoid $B_{M_{p}}(0, C \delta)$ in $M$ will emanate a distance of roughly $\delta^{2}$ from $M$. However, the hull of holomorphy of an ellipsoid which is much thinner in the $z_{3}$ direction will also emanate a distance of roughly $\delta^{2}$ from $M$. Thus, our results are most interesting in $C^{2}$. In fact, we shall reduce the higher dimensional case to the case of $C^{2}$ by a slicing technique.

Our paper is organized as follows. Precise definitions and statements of results are given in $\S 2$. This includes definitions of the nonisotropic balls and the quantity $\Lambda_{M}(p, \delta)$. Results about these balls were merely announced in [NSW1], and in $\S 3$, we give complete proofs of needed results and some equivalent descriptions of these nonisotropic balls. We also reformulate our main results in terms of these equivalent definitions (Theorem 3.18). The proof of our theorem on the hull of holomorphy is then completed in $\S \S 4$ and 5. The proof of our estimate for plurisubharmonic functions is given in $\S 6$.

We would like to thank Steve Wainger for several stimulating conversations about the possibility of obtaining results for the finite type case without the hypothesis of pseudoconvexity. We would also like to thank the referee for his thorough reading of the manuscript. The referee made many suggestions which simplified the proof considerably. 


\section{NOTATION AND STATEMENT OF RESULTS}

First let us fix notation. Let $U \subset C^{n}$ be an open set and let $M \subset U$ be a smooth real hypersurface given by

$$
M=\{z \in U ; r(z)=0\},
$$

where $r \in C^{\infty}(U)$ and $\nabla r(z) \neq 0$ when $r(z)=0$. We let

$$
U^{+}=\{z \in U ; r(z)>0\}, \quad U^{-}=\{z \in U ; r(z)<0\} .
$$

We let $T(M)$ be the real tangent bundle to $M$, and we let $H(M)$ be the holomorphic ( $J$-invariant) subbundle of $T(M)$. We let $H^{C}(M)=H^{1,0}(M)+$ $H^{0,1}(M)$ be the complexification of $H(M)$ in the usual way. Note that for $p \in$ $M$, the fibers over $p$ have the following dimensions: $\operatorname{dim}_{C}\left(H_{p}^{C}(M)\right)=2 n-2$ and $\operatorname{dim}_{C} H_{p}^{1,0}(M)=n-1=\operatorname{dim}_{C} H_{p}^{0,1}(M)$.

Near a fixed point $p_{0} \in M$ we can choose a local set of generators $L_{1}, \ldots, L_{n-1}$ for $H^{1,0}(M)$ (so $\bar{L}_{1}, \ldots, \bar{L}_{n-1}$ locally generate $H^{0,1}(M)$ ). We can also locally choose a vector field $T$ which is transverse to $H^{C}(M)$, so the complexified tangent bundle of $M$ is generated near $p_{0}$ by the $L$ 's, the $\bar{L}$ 's, and $T$.

For convenience, let $L_{j+n-1}=\bar{L}_{j}$ for $j=1, \ldots, n-1$. Now for $p \in M$ near $p_{0}$ and $1 \leq i_{1}, \ldots, i_{j} \leq 2 n-2$ define $\lambda_{i_{1}, \ldots, i_{j}}(p)$ by the equation

$$
\left[L_{i_{j}},\left[\ldots\left[L_{i_{2}}, L_{i_{1}}\right] \ldots\right]\right]_{p}=\lambda_{i_{1}, \ldots, i_{j}}(p) T_{p} \quad \bmod H_{p}^{C}(M) .
$$

We let

$$
\Lambda_{j}(p)=\sum\left|\lambda_{i_{1} \cdots i_{k}}(p)\right|,
$$

where $j \geq 2$ and the sum runs over all $i_{1}, \ldots, i_{k}$ with $k \leq j$ and $1 \leq$ $i_{1}, \ldots, i_{k} \leq 2 n-2$. Note that when $j=2$, the matrix $\left\{\lambda_{i_{1}, i_{2}}(p)\right\}, i_{1}=$ $1, \ldots, n-1, i_{2}=n, \ldots, 2 n-2$, is the usual Levi form matrix of $M$ at $p$.

We now suppose $M$ has finite type $m_{0}$. Recall (see Kohn [K] or Bloom and Graham [BG]) that a point $p \in M$ has type $m$ if for every choice of vector fields $L_{1}, \ldots, L_{n-1}$ and $T$, all commutators of the $L$ 's and $\bar{L}$ 's at $p$ of length less than $m$ have zero $T$ component, and there is some commutator among the $L$ 's and $\bar{L}$ 's at $p$ of length $m$ which has a nonzero $T$ component.

We now define our size function $\Lambda_{M}(p, \delta)$ for $p \in M$ near $p_{0}$ and $\delta>0$ by

$$
\Lambda_{M}(p, \delta)=\sum_{j=2}^{m_{0}} \Lambda_{j}(p) \delta^{j} .
$$

Note that this function $\Lambda_{M}$ depends on the choice of vector fields $L_{1}, \ldots, L_{n-1}$ and $T$. However, a different choice of $L$ 's and $T$ will result in a new function $\widetilde{\Lambda}_{M}$ and the ratio $\Lambda_{M} / \tilde{\Lambda}_{M}$ will be bounded and bounded away from zero near $p_{0}$. Thus, although we will generally work locally near a fixed point $p_{0} \in M$, we can patch the locally defined functions together to obtain a function $\Lambda_{M}(p, \delta)$ defined for all $p \in M$ and $\delta>0$. The finite type hypothesis then implies that 
for every compact set $K$ in $M$ there are positive constants $C_{1}$ and $C_{2}$ so that $p \in K$ and $0 \leq \delta \leq 1$ :

$$
C_{1} \delta^{m_{0}} \leq \Lambda_{M}(p, \delta) \leq C_{2} \delta^{2} .
$$

Let us write $L_{j}=X_{j}+i Y_{j}$ for $1 \leq j \leq n-1$, where $X_{j}$ and $Y_{j}$ are real vector fields in $T(M)$.

Definition 2.2. For $p \in M, p$ near $p_{0}$, and $\delta>0$ let

$$
\begin{array}{r}
B_{M}(p, \delta)=\left\{q \in M ; q=\exp \left[\sum_{j=1}^{n-1} \alpha_{j} X_{j}+\beta_{j} Y_{j}+\gamma T\right](p)\right. \\
\text { where } \alpha_{j}, \beta_{j}, \gamma \in R \text {, with }\left|\alpha_{j}\right|<\delta, \\
\left.\quad\left|\beta_{j}\right|<\delta,|\gamma|<\Lambda_{M}(p, \delta) \text { for } 1 \leq j \leq n-1\right\} .
\end{array}
$$

Here, exp refers to the usual exponential map. As mentioned in the introduction, these balls are essentially twisted ellipsoids of radius $\delta$ in the complex tangential directions, and radius $\Lambda_{M}(p, \delta)$ in the direction $T$. A different choice of vector fields will result in different but comparable balls.

We call a smooth map $\pi: U \rightarrow M$ a projection if for every $p \in M, \pi(p)=$ $p$ and $\left\{\pi^{-1}(p)\right\}$ is a smooth one-dimensional submanifold of $U$ which is transverse to $M$.

The local hull of holomorphy of $B_{M}(p, \delta)$ will be described in terms of certain open set $V_{\mu}^{ \pm}$in the general case and $O_{C}(p, \delta)$ in the case $M$ bounds a pseudoconvex domain. In the definition and the theorems which follow, we shall assume that the defining function $r$ is chosen so that if $M$ bounds a pseudoconvex domain, then $U^{-}$is pseudoconvex.

Definition 2.3. Let

(a) $V_{\mu}^{ \pm}(p, \delta)=\left\{q \in U^{ \pm} ; \pi(q) \in B_{M}(p, \delta)\right.$ and $\left.|\pi(q)-q|<\mu \Lambda_{M}(p, \delta)\right\}$.

For $q \in U^{-}$with $\pi(q) \in B_{M}(p, \delta)$, let

and let

$$
\delta_{q}=\delta_{q, p}=\sup \left\{t>0 ; B_{M}(\pi(q), t) \subset B_{M}(p, \delta) p\right\}
$$

(b) $O_{\mu}(p, \delta)=\left\{q \in U^{-} ; \pi(q) \in B_{M}(p, \delta)\right.$ and $\left.|\pi(q)-q|<\mu \Lambda_{M}\left(\pi(q), \delta_{q}\right)\right\}$.

Here $|\cdot|$ refers to the Euclidean distance in $C^{n}$.

Our main result is the following.

Theorem 2.4. (a) Suppose $M \subset U \subset C^{n}$ is a smooth real hypersurface of finite type $m_{0}$, and let $K \subset \subset M$ be compact. There are an open set $U^{\prime} \subset U$ with $K \subset \subset U^{\prime}$, a projection $\pi: U^{\prime} \rightarrow M \cap U^{\prime}$, and constants $\mu_{0}, \varepsilon_{0}, \delta_{0}>0$ so that if $p \in K$ and $0<\delta \leq \delta_{0}$, then the hull of holomorphy of the set $B_{M}(p, \delta)$ contains either $V_{\mu_{0}}^{+}\left(p, \varepsilon_{0} \delta\right)$ or $V_{\mu_{0}}^{-}\left(p, \varepsilon_{0} \delta\right)$ (or both).

(b) If in addition $M$ is the smooth boundary of a pseudoconvex domain $D \quad\left(=U^{-}\right)$of finite type, then there are constants $\delta_{0}, C, \mu_{0}>0$ so that if 
$0 \leq \delta \leq \delta_{0}$, then the hull of holomorphy of the set $B_{M}(p, \delta)$ contains the open set $O_{\mu_{0}}(p, \delta)$.

As mentioned in the introductior, our approach to this theorem involves analytic discs. An analytic disc is a continuous map $A$ from the closed unit disc in $C$ to $C^{n}$ which is holomorphic on the open unit disc.

In order to prove Theorem 2.4(b) for example, it suffices to show that each point $q \in O_{\mu_{0}}(p, \delta)$ is the center of an analytic disc $A$ whose entire image lies in $\pi^{-1}\left\{B_{M}(p, \delta)\right\}$ and whose boundary lies in the set $\pi^{-1}\left\{B_{M}(p, \delta)\right\} \cap U^{+}$, i.e., we want the center of the disc $A$ (i.e., the point $q$ ) and the boundary of the disc $A$ to lie on opposite sides of $M$. Once this is accomplished, the point $q$ must lie in the hull of $B_{M}(p, \delta)$ in view of the analyticity of $A$ and the maximum principle applied to the set $\left\{\zeta\right.$ in the unit disc; $\left.A(\zeta) \in U^{-}\right\}$.

Therefore, Theorem 2.4 reduces to

Theorem 2.5. With all the assumptions and notation of Theorem 2.4 , we have

(a) Each point in $V_{\mu_{0}}^{+}\left(p, \varepsilon_{0} \delta\right)$ (or in $V_{\mu_{0}}^{-}\left(p, \varepsilon_{0} \delta\right)$ ) is the center of an analytic disc whose image lies in $\pi^{-1}\left\{B_{M}(p, \delta)\right\}$ and whose boundary lies in $\pi^{-1}\left\{B_{M}(p, \delta)\right\} \cap U^{-}$(or in $\left.\pi^{-1}\left\{B_{M}(p, \delta)\right\} \cap U^{+}\right)$.

(b) If $M$ bounds a pseudoconvex domain, each point of $O_{\mu_{0}}(p, \delta)$ is the center of an analytic disc whose image lies in $\pi^{-1}\left\{B_{M}(p, \delta)\right\}$ and whose boundary lies in $\pi^{-1}\left\{B_{M}(p, \delta)\right\} \cap U^{+}$.

Theorem 2.5 together with the $\mathbf{C R}$ approximation theorem of Baouendi and Treves [BT] and the maximum principle yield the following $\mathbf{C R}$ extension result: Continuous CR functions on $B_{M}(p, \delta)$ extend to holomorphic functions on $V_{\mu_{0}}^{+}\left(p, \varepsilon_{0} \delta\right)$ or $V_{\mu_{0}}^{-}\left(p, \varepsilon_{0} \delta\right)$ under the assumptions given in part (a) in Theorem 2.4 , and to $O_{\mu_{0}}(p, \delta)$ under the assumptions given in part (b) of Theorem 2.4. The above mentioned theorem of Baouendi and Treves as stated in their paper is a local theorem about a fixed point. However, a careful look at the proof shows that the size of the neighborhood on which $\mathbf{C R}$ functions can be approximated can be made independent of the point lying in some fixed compact set.

Finally, we state our theorem on estimates for plurisubharmonic functions.

Theorem 2.6. Let $M$ be the smooth boundary of a bounded pseudoconvex domain of finite type. Then there is a projection $\pi: D \cap U \rightarrow M$ and there are constants $\delta_{0}, C, A>0$ with the following property. Let $u$ be a continuous function on $\bar{D}$ which is plurisubharmonic on $D$ and let $z \in D \cap U$ with $|z-\pi(z)|=C \Lambda_{M}(\pi(z), \delta)$ and $0<\delta \leq \delta_{0}$. Then

$$
u(z) \leq \frac{A}{|B|} \int_{\zeta \in B}|u(\zeta)| d \sigma(z),
$$

where $B=B_{M}(\pi(z), \delta)$. 
Here $|B|$ refers to the Lebesgue measure of the ball $B$. Note that the constants $C, A$, and $\delta_{0}$ are independent of the plurisubharmonic function $u$, the point $z$, and $0<\delta \leq \delta_{0}$.

\section{EQUiVALENT FORMULATION OF THE SIZE FUNCTION AND BALLS}

It will be convenient to have a more computational formula for $\Lambda_{M}(p, \delta)$ and the balls $B_{M}(p, \delta)$ in terms of defining functions for $M$. In turn, this formula will be simplified if the defining functions for $M$ have a nice form. To this end, let $K \subset \subset M$ be compact. We define a family of local biholomorphisms $H_{p}: C^{n} \rightarrow C^{n}$ indexed by $p \in M$ and which depend smoothly on $p$. We require the following properties on the family of $H_{p}$.

(1) $H_{p}(0)=p$.

(2) There is a neighborhood $\widetilde{U}$ of 0 in $C^{n}$ such that for each $p \in M$ there is a neighborhood $U_{p}$ in $C^{n}$ of $p$ with $H_{p}: \widetilde{U} \rightarrow U_{p}$ a biholomorphism.

(3) Suppose $D=\left\{q \in C^{n} ; \rho(q)<0\right\}$ and let $\rho^{p}=\rho \circ H_{p}$. With coordinates $(z, w) \in C \times C^{n-1}, z=x+i y$ we require $\rho^{p}(x+i y, w)=$ $h^{p}(y, w)-x$ where $h^{p}: R \times C^{n-1} \rightarrow R$ is smooth and has no pure terms, i.e.,

$$
\frac{\partial^{|\alpha|} h^{p}(0)}{\partial w^{\alpha}}=0 \text { for } 0 \leq|\alpha| \leq m_{0}
$$

(recall $m_{0}$ is the maximum type of any point in $M$ ).

(4) The family $\left\{H_{p} ; p \in K\right\}$ is a bounded set in $C^{m_{0}+2}(\widetilde{U})$.

Thus $H_{p}^{-1}$ takes $p$ to 0 and takes $M \cap U_{p}$ to a hypersurface which we will denote by $M_{p}$ and which is graphed over its tangent space at the origin with a graphing function which has no pure terms. The construction of the $H_{p}$ is straightforward and will be omitted.

Clearly, there is a constant $C>0$ such that if $x \in R$ with $|x|<C$, then $(x+i 0,0) \in \widetilde{U}$. The set $U=\left\{H_{p}(x+i 0,0) ; p \in M,|x|<C\right\}$ is therefore an open neighborhood of $M$ in $C^{n}$ and our desired projection map $\pi: U \cap D \rightarrow M$ for Theorems 2.4, 2.5, and 2.6 and can be constructed so that for each $p \in M$, $\pi^{-1}(p)=H_{p}\{(x+i 0,0) ;|x|<C\}$. Since $M$ and $M_{p}$ are biholomorphically related, clearly $\Lambda_{M}(p, \delta) \approx \Lambda_{M_{p}}(0, \delta)$, where here and below $A \approx B$ means that there are constants $0<C_{1} \leq C_{2}<\infty$ which are independent of $p$ and $\delta$ so that

$$
C_{1} A \leq B \leq C_{2} A .
$$

Note that this means that the sets $H_{p}\left\{B_{M_{p}}(0, \delta)\right\}$ and $B_{M}(p, \delta)$ are comparable.

We shall now derive an equivalent formula for $\Lambda_{M_{p}}(0, \delta)$. 
Definition 3.1. Suppose $h: R \times C^{n-1} \rightarrow R$ is smooth. For $2 \leq m<\infty$ we define

$$
\Lambda_{m}^{h}(\delta)=\sum_{j=2}^{m} \sum_{\substack{\alpha=\left(\alpha_{1}, \ldots, \alpha_{n-1}\right) \\ \beta=\left(\beta_{1}, \ldots, \beta_{n-1}\right) \\|\alpha|+|\beta|=j}}\left|\frac{\partial^{j} h(0)}{\partial w^{\alpha} \partial \bar{w}^{\beta}}\right| \delta^{j} .
$$

Lemma 3.2. Suppose $M_{p}$ and $h^{p}$ are defined as above. Then

$$
\Lambda_{M}(p, \delta) \approx \Lambda_{M_{p}}(0, \delta) \approx \Lambda_{m_{0}}^{h^{p}}(\delta)
$$

where $m_{0}$ is the maximum type of any point on $M$.

Proof. As already mentioned, $\Lambda_{M}(p, \delta) \approx \Lambda_{M_{p}}(0, \delta)$ holds because $M_{p}$ is a biholomorphic image of $M$.

The definition of $\lambda_{i_{1}, \ldots, i_{j}}$ given in $\S 2$ depends on the choice of vector fields for the tangent space of $M_{p}$ and a different choice of vector fields gives an equivalent $\lambda_{i_{1}, \ldots, i_{j}}$. Thus we shall make a fixed choice of vector fields that we shall use. With $z=x+i y$, a basis for the complexified holomorphic tangent bundle is given by $\widetilde{L}_{1}, \ldots, \widetilde{L}_{2 n-2}$, where

$$
\begin{gathered}
\widetilde{L}_{j}=\frac{\partial}{\partial w_{j}}+2 \frac{h_{u_{j}}^{p}}{\left(1+i h_{y}^{p}\right)} \frac{\partial}{\partial z} \text { for } 1 \leq j \leq n-1, \\
\widetilde{L}_{j+n-1}=\bar{L}_{j} \text { for } 1 \leq j \leq n-1 .
\end{gathered}
$$

It will be covenient to work on the tangent space of $M_{p}$ at the origin which can be identified with $R \times C^{n-1}$. Let $\tilde{\pi}: M_{p} \rightarrow R \times C^{n-1}$ be the usual projection map, i.e., $\tilde{\pi}\left(h^{p}(y, w)+i y, w\right)=(y, w) \in R \times C^{n-1}$. Let $L_{j}=d \tilde{\pi}\left\{\widetilde{L}_{j}\right\}$ for $1 \leq j \leq 2 n-2$. Then

$$
\begin{gathered}
L_{j}=\frac{\partial}{\partial w_{j}}-\frac{i h_{w_{j}}^{p}}{\left(1+i h_{y}^{p}\right)} \frac{\partial}{\partial y} \text { for } 1 \leq j \leq n-1, \\
L_{j+n-1}=\bar{L}_{j} \text { for } 1 \leq j \leq n-1 .
\end{gathered}
$$

The transverse vector field will be $T=\frac{\partial}{\partial y}$. To conform with the notation above for the $L$ 's, we let

$$
\frac{\partial}{\partial w_{j+n-1}}=\frac{\partial}{\partial \bar{w}_{j}} \text { for } 1 \leq j \leq n-1 .
$$

With the above convention, we may rewrite

$$
\Lambda_{m}^{h^{p}}(\delta)=\sum_{j=2}^{m} \sum_{i_{1}, \ldots, i_{j}}^{2 n-2}\left|\frac{\partial h^{p}(0)}{\partial w_{i_{1}}, \ldots, \partial w_{i_{j}}}\right| \delta^{j}
$$

Since $h^{p}$ has no pure terms, nonvanishing terms in the above sum occur only when one of the above indices is restricted to $1, \ldots, n-1$ (the unconjugated 
variable indices) and another (different) index is restricted to $n, \ldots, 2 n-2$ (the conjugated variable indices). Therefore we may write

$$
\Lambda_{m}^{h^{p}}(\delta)=2 \sum_{j=2}^{m} \sum_{i_{1}=1}^{n-1} \sum_{i_{2}=n}^{2 n-2} \sum_{i_{3}, \ldots, i_{j}=1}^{2 n-2}\left|\frac{\partial^{j} h^{p}(0)}{\partial w_{i_{1}}, \ldots, \partial w_{i_{j}}}\right| \delta^{j} .
$$

Now we shall unravel the $\lambda_{i_{1}, \ldots, i_{j}}$ where on $R \times C^{n-1}$ the $\lambda_{i_{1}, \ldots, i_{j}}$ is given by

$$
\left[L_{i_{j}},\left[\ldots,\left[L_{i_{2}}, L_{i_{1}}\right], \ldots,\right]\right]_{q}=\lambda_{i_{1}, \ldots, i_{j}}(q) T_{q} \quad \bmod H_{q}^{C}\left(M_{p}\right)
$$

for $q=(y, w) \in R \times C^{n-1}$.

We define $I_{j}$ to be the ideal spanned over $C^{\infty}\left(R \times C^{n-1}\right)$ by

$$
\left\{\lambda_{i_{1}, \ldots, i_{k}} ; 1 \leq i_{1}, \ldots, i_{k} \leq 2 n-2 \text { for } k \leq j\right\},
$$

and $W_{j}$ to be the ideal spanned over $C^{\infty}\left(R \times C^{n-1}\right)$ by

$$
\left\{\frac{\partial^{k} h^{p}}{\partial w_{i_{1}}, \ldots, \partial w_{i_{k}}} ; 1 \leq i_{1}, \ldots, i_{k} \leq 2 n-2 \text { and } k \leq j\right\} \text {. }
$$

Lemma 3.4. For $2 \leq j \leq m_{0}$

(a)

$$
\lambda_{i_{1}, \ldots, i_{j}}=L_{i_{j}}\left\{L_{i_{j-1}}, \ldots, L_{i_{3}}\left\{\lambda_{i_{1} i_{2}}\right\} \ldots\right\} \quad \bmod I_{j-1} .
$$

(b) If $1 \leq i_{1} \leq n-1$ and $n \leq i_{2} \leq 2 n-2$, or $1 \leq i_{2} \leq n-1$ and $n \leq i_{1} \leq 2 n-2$, then

$$
L_{i_{j}} \cdots L_{i_{3}}\left\{\lambda_{i_{1} i_{2}}\right\}=g \cdot \frac{\partial^{j} h^{p}}{\partial w_{i_{1}} \cdots \partial w_{i_{j}}} \quad \bmod W_{j-1},
$$

where $g$ is a smooth function with $|g(0)|=2$.

(c) If $1 \leq i_{1}, i_{2} \leq n-1$ or $n \leq i_{1}, i_{2} \leq 2 n-2$, then

$$
L_{i_{1}} \cdots L_{i_{3}}\left\{\lambda_{i_{1} i_{2}}\right\} \in W_{j-1} \text {. }
$$

Proof. Part (a) is easily proved by induction on $j$. For part (b), we first compute $\lambda_{i_{1} i_{2}}$ in the case $1 \leq i_{1} \leq n-1$ and $n \leq i_{2} \leq 2 n-2$. After an easy computation using the formulas for the $L_{j}$, we obtain

$$
\lambda_{i_{1} i_{2}}=h_{w_{i_{1}} w_{i_{2}}}^{p} \cdot\left(\frac{-2 i}{1+\left(h_{y}^{p}\right)^{2}}\right) \bmod W_{1} .
$$

We let $g$ be the term in the parentheses on the right. The above formula establishes part (b) for this case when $j=2$ because $h_{y}^{p}(0)=0$. Using the formula for the $L_{j}$, it is clear that if $f \in W_{k}$, then $L_{j} f \in W_{k+1}$. Therefore, applying $L_{i_{j}}, \ldots, L_{i_{3}}$ to the above equation establishes part (b) for $j \geq 3$. 
Since $\overline{\lambda_{i_{1} i_{2}}}=\lambda_{i_{2}, i_{1}}$, clearly the same arguments above establish the case when $1 \leq i_{2} \leq n-1$ and $n \leq i_{1} \leq 2 n-2$. This completes part (b).

For part (c), if, say, $1 \leq i_{1}, i_{2} \leq n-1$, then

$$
\lambda_{i_{1} i_{2}}=\frac{\partial}{\partial w_{i_{2}}}\left(\frac{-i h_{w_{i_{1}}}^{p}}{\left(1+i h_{y}^{p}\right)}\right)+\frac{\partial}{\partial w_{i_{1}}}\left(\frac{i h_{{i_{2}}_{i_{2}}}^{p}}{\left(1+i h_{y}^{p}\right)}\right)
$$

which clearly lies in $W_{1}$. The proof of part (c) then follows easily from the formulas for the $L_{j}$.

Now we can complete the proof of Lemma 3.2. We shall show by induction on $m, 2 \leq m \leq m_{0}$, that

$$
\sum_{j=2}^{m} \sum_{i_{1}, \ldots, i_{j}=1}^{2 n-2}\left|\lambda_{i_{1}, \ldots, i_{j}}(0)\right| \delta^{j} \approx \Lambda_{m}^{h^{p}}(\delta) .
$$

When $m=2$, both sides of (3.6) are equivalent to the usual Levi form (multiplied by $\delta^{2}$ ) and so the proof is easy in this case. So we assume (3.6) for $m-1$ and prove it for $m$. We have

$$
\sum_{j=2}^{m} \sum_{i_{1}, \ldots, i_{i}}\left|\lambda_{i_{1}, \ldots, i_{j}}(0)\right| \delta^{j} \approx \sum_{i_{1}, \ldots, i_{m}}\left|\lambda_{i_{1}, \ldots, i_{m}}(0)\right| \delta^{m}+\Lambda_{m-1}^{h^{p}}(\delta)
$$

by the induction hypothesis. Note from Lemma 3.4 with $j=m$, that $\lambda_{i_{1}, \ldots, i_{m}} \in$ $W_{m-1}$ if $1 \leq i_{1}, i_{2} \leq n-1$ or $n \leq i_{1}, i_{2} \leq 2 n-2$. Thus

$$
\left|\lambda_{i_{1}, \ldots, i_{m}}(0)\right| \delta^{m} \leq C \delta \Lambda_{m-1}^{h^{\prime}}(\delta)
$$

if $1 \leq i_{1}, i_{2} \leq n-1$ or $n \leq i_{1}, i_{2} \leq 2 n-2$. Combining this with Lemma 3.4 for the case $1 \leq i_{1} \leq n-1, n \leq i_{2} \leq 2 n-2$ or the case $1 \leq i_{2} \leq n-1$, $n \leq i_{1} \leq 2 n-2$ we obtain

$$
\begin{aligned}
& \sum_{i_{1}, \ldots, i_{m}=1}^{2 n-2}\left|\lambda_{i_{1}, \ldots, i_{m}}(0)\right| \delta^{m}-2 \sum_{i_{1}=1}^{n-1} \sum_{i_{2}=n}^{2 n-2} \sum_{i_{3}, \ldots, i_{m}=1}^{2 n-2}\left|\frac{\partial^{m} h^{p}(0)}{\partial w_{i_{1}}, \ldots, w_{i_{m}}}\right| \delta^{m} \\
& \leq C \delta \Lambda_{m-1}^{h^{\prime}}(\delta)
\end{aligned}
$$

for some uniform constant $C>0$. The right side of the above inequality results from the " $\bmod I_{m-1}$ " terms in part (a) of Lemma 3.4 and the " $\bmod W_{m-1}$ " terms in part (b) in Lemma 3.4 and the induction hypothesis. In view of (3.3), inequalities (3.8) and (3.7) easily establish (3.6) provided $\delta$ is suitably restricted so that the right-hand side of (3.8) can be absorbed into the right side of (3.6). This completes the proof of Lemma 3.2.

The following easy lemma is used throughout much of the rest of this paper to absorb higher order error terms in $\delta$. 
Lemma 3.9. Given a compact set $K \subset M$, there is a constant $C>0$ which is independent of $p \in K$ and $\delta>0$ so that if $m \geq m_{0}$ then

$$
\delta^{m} \leq C \delta^{m-m_{0}} \Lambda_{M_{p}}(0, \delta) .
$$

Proof. Since $M$ is a compact hypersurface of type $m_{0}<\infty$, clearly there is a constant $C>0$ such that

$$
\sum_{j=2}^{m_{0}} \sum_{|\alpha|+|\beta|=j}\left|\frac{\partial^{j} h^{p}(0)}{\partial w^{\alpha} \partial \bar{w}^{\beta}}\right| \geq C
$$

for all $p \in M$. Let $N$ be the number of terms in the above sum. For each $p \in M$, there must be a choice of $\alpha$ and $\beta$ (depending on $p$ ) with

$$
\left|\frac{\partial^{|\alpha|+|\beta|} h^{p}(0)}{\partial w^{\alpha} \partial \bar{w}^{\beta}}\right| \geq \frac{C}{N} \text {. }
$$

We then have for $m \geq m_{0}$

$$
\begin{aligned}
\delta^{m} & \leq \delta^{m-(|\alpha|+|\beta|)} \frac{N}{C}\left|\frac{\partial^{|\alpha|+|\beta|} h^{p}(0)}{\partial w^{\alpha} \partial \bar{w}^{\beta}}\right| \delta^{|\alpha|+|\beta|} \\
& \leq \frac{N}{C} \delta^{m-m_{0}} \Lambda_{m_{0}}^{h^{p}}(\delta)
\end{aligned}
$$

which establishes our lemma after using Lemma 3.2.

Now we shall give an alternative description of the ball $B_{M_{p}}(0, \delta)$ in terms of the graph of $h^{p}$ over a rectangle in $R \times C^{n-1}$ of width $\delta$ in the $C^{n-1}$ direction and width $\Lambda_{M_{p}}(0, \delta)$ in the $y$ direction. Let

$$
\widetilde{B}_{M_{p}}(0, \delta)=\left\{\left(h^{p}(y, w)+i y, w\right) ;|w|<\delta \text { and }|y|<\Lambda_{M_{p}}(0, \delta)\right\} .
$$

Lemma 3.10. The balls $B_{M_{p}}(0, \delta)$ and $\widetilde{B}_{M_{p}}(0, \delta)$ are comparable. That is, there are uniform constants $0<C_{1} \leq C_{2}<\infty$ which are independent of $p$ and $\delta$ and there is a constant $\delta_{0}$ so that if $0 \leq \delta \leq \delta_{0}$, then

$$
B_{M_{p}}\left(0, C_{1} \delta\right) \subset \widetilde{B}_{M_{p}}(0, \delta) \subset B_{M_{p}}\left(0, C_{2} \delta\right) .
$$

Proof. For $1 \leq j \leq n-1$, we let $X_{j}$ be the real part and $Y_{j}$ the imaginary part of the vector fields $L_{j}$ defined in the proof of Lemma 3.2. Thus

$$
\begin{aligned}
& X_{j}=\frac{1}{2}\left(\frac{\partial}{\partial u_{j}}-\frac{h_{v_{j}}^{p}+h_{y}^{p} h_{u_{j}}^{p}}{1+\left(h_{y}^{p}\right)^{2}} \cdot \frac{\partial}{\partial y}\right), \\
& Y_{j}=\frac{1}{2}\left(\frac{-\partial}{\partial v_{j}}-\frac{h_{u_{j}}^{p}-h_{y}^{p} h_{v_{j}}^{p}}{1+\left(h_{h}^{p}\right)^{2}} \cdot \frac{\partial}{\partial y}\right),
\end{aligned}
$$

where $w_{j}=u_{j}+i v_{j}, 1 \leq j \leq n-1$. As in Lemma 3.4, we let

$$
T=\frac{\partial}{\partial y} \text {. }
$$


Recall that $\tilde{\pi}: M_{p} \rightarrow R \times C^{n-1}$ denotes the standard projection and that the vector fields $X_{j}, Y_{j}$, and $T$ are images under $d \pi$ of vector fields defined on $M_{p}$. Since $\tilde{\pi} \circ \exp =\exp \circ d \tilde{\pi}$, it suffices to show that the sets

$$
R(\delta)=\left\{(y, w) \in R \times C^{n-1} ;|w|<\delta,|y|<\Lambda_{M_{p}}(0, \delta)\right\}
$$

and

$$
\begin{array}{r}
B(\delta)=\left\{q \times R \times C^{n-1} ; q=\exp \left(\sum_{j=1}^{n-1} \alpha_{j} X_{j}+\beta_{j} Y_{j}+\gamma T\right)\right. \\
\text { where } \left.\alpha_{j}, \beta_{j}, \gamma \in R \text { with }\left|\alpha_{j}\right|,\left|\beta_{j}\right|<\delta,|\gamma|<\Lambda_{M_{p}}(0, \delta)\right\}
\end{array}
$$

are comparable.

By the definition of the exponential map, if $q \in B(\delta)$, then $q=\varphi(1)$, where $\varphi:[0,1] \rightarrow R \times C^{n-1}$ is a smooth map with

$$
\varphi^{\prime}(t)=\sum_{j=1}^{n-1}\left(\left.\alpha_{j} X_{j}\right|_{\varphi(t)}+\left.\beta_{j} Y_{j}\right|_{\varphi(t)}+\left.\gamma T\right|_{\varphi(t)} ;\right.
$$

here $\left|\alpha_{j}\right|,\left|\beta_{j}\right|<\delta$ and $|\gamma|<\Lambda_{M_{p}}(0, \delta)$. By writing out $\varphi$ in components as $\varphi=\left(\eta, \xi_{1}+i \psi_{1}, \ldots, \xi_{n-1}+i \psi_{n-1}\right)$, where $\xi_{j}, \psi_{j}$, and $\eta$ are all smooth real valued functions, and then using the component descriptions of $X_{j}, Y_{j}$, and $T$ given in (3.11a, b, c), we obtain

$$
\begin{gathered}
\xi_{j}^{\prime}(t)=\alpha_{j} / 2, \quad 1 \leq j \leq n-1, \\
\psi_{j}^{\prime}(t)=-\beta_{j} / 2, \quad 1 \leq j \leq n-1, \\
\eta^{\prime}(t)=\gamma+F(\varphi(t)),
\end{gathered}
$$

where

$$
F=-\frac{1}{2} \sum_{j=1}^{n-1} \frac{\alpha_{j}\left(h_{v_{j}}^{p}+h_{y}^{p} h_{u_{j}}^{p}\right)+\beta_{j}\left(h_{u_{j}}^{p}-h_{y}^{p} h_{v_{j}}^{p}\right)}{1+\left(h_{y}^{p}\right)^{2}} .
$$

Integrating the above equations yields

$$
\begin{gathered}
\xi_{j}(t)=\alpha_{j} t / 2, \\
\psi_{j}(t)=-\beta_{j} t / 2, \\
\eta(t)=\gamma t+\int_{0}^{t} F\left(\eta(s), \frac{1}{2}(\alpha-i \beta) s\right) d s,
\end{gathered}
$$

where we have written $\alpha=\left(\alpha_{1}, \ldots, \alpha_{n-1}\right) \in R^{n-1}$ and $\beta=\left(\beta_{1}, \ldots, \beta_{n-1}\right) \in$ $R^{n-1}$. Since $h_{y}^{p}(0,0)=0$, the denominator of $F$ stays bounded away from zero provided $\delta$ is kept sufficiently small. So, we examine the Taylor expansion of the numerator of $F$ of order $m_{0}$ in powers of $\eta$ and $w=\frac{1}{2}(\alpha-i \beta) s$ and 
$\bar{w}=\frac{1}{2}(\alpha+i \beta) s$, keeping in mind that $|\alpha|,|\beta|$ are less than $\delta,|\eta| \leq \Lambda_{M_{p}}(0, \delta)$, and $0 \leq s \leq 1$. Note that the Taylor remainder of order $m_{0}+1$ can be made negligible compared to $\Lambda_{M_{p}}(0, \delta)$ by suitably restricting $\delta$ in view of Lemma 3.9. We recall that $\nabla h(0)=0$, so there is no constant term in the expansion. Any term involving a factor of $\eta$ will be dominated by $\delta\|\eta\|$, where $\|\eta\|=\sup _{0 \leq t \leq 1}|\eta(t)|$. The factor of $\delta$ comes from the factors of $\alpha_{j}$ and $\beta_{j}$ in the numerator of $F$ which stay less than $\delta$ in absolute value. The terms in the expansion of $h_{u_{j}}^{p}$ and $h_{v_{j}}^{p}$ which only involve $w$ and $\bar{w}$ are all dominated in absolute value by some constant multiple of $\Lambda_{M_{p}}(0, \delta)$ in view of Lemma 3.2 (recall that the expansion of $h^{p}$ involves no pure terms). In addition, $|\gamma|<\Lambda_{M_{p}}(0, \delta)$. Therefore, after taking the supremum of both sides of $(3.12 \mathrm{c})$ for $0 \leq t \leq 1$, we obtain

$$
\|\eta\| \leq C\left(\delta\|\eta\|+\Lambda_{M_{p}}(0, \delta)\right) .
$$

One can then easily absorb the term involving $\delta\|\eta\|$ appearing on the right side of the above inequality by suitably restricting $\delta$. In particular, $|\eta(1)| \leq$ $C \Lambda_{M_{p}}(0, \delta)$. In addition, clearly $)\left|\xi_{j}(1)\right|=\frac{1}{2}\left|\alpha_{j}\right|<\delta / 2$ and $\left|\psi_{j}(1)\right|=\frac{1}{2}\left|\beta_{j}\right|<$ $\delta / 2$. Thus

$$
B(\delta) \subset R(C \delta)
$$

for some uniform positive constant $C$.

To establish the other inclusion, we fix a positive constant $\varepsilon$ which will be chosen later. Suppose that $(y, w) \in R(\varepsilon \delta)$. We wish to find the function $\varphi$ whose components are given in (3.12) so that $\varphi(1)=(y, w)$. If $\alpha_{j}=2 \operatorname{Re} w_{j}$ and $\beta_{j}=-2 \operatorname{Im} w_{j}$, then $\xi_{j}(1)+i \psi_{j}(1)=w_{j}$ and $\left|\alpha_{j}\right|,\left|\beta_{j}\right| \leq 2 \varepsilon \delta$ for $1 \leq$ $j \leq n-1$. With this choice of $\alpha$ and $\beta$, one can then repeat the analysis following $(3.12 \mathrm{c})$ to show

$$
\|\eta\| \leq C \varepsilon \Lambda_{M_{p}}(0, \delta) .
$$

The analysis following (3.12c) together with the above estimate on $\eta$ show

$$
\left|F\left(\eta(s), \frac{1}{2}(\alpha-i \beta) s\right)\right| \leq C \varepsilon \Lambda_{M_{p}}(0, \delta) \text { for } 0 \leq s \leq 1 .
$$

Therefore from $(3.12 \mathrm{c})$, we have

$$
\eta(t) \geq t(\gamma-C) \varepsilon \Lambda_{M_{p}}(0, \delta)
$$

where $C$ is some uniform positive constant. We now restrict $\varepsilon$ so that $0<$ $\varepsilon<\min \left\{\frac{1}{2}, \frac{1}{2} / C\right\}$. Since $\eta(0)=0$, it is now clear from the above inequality that if $|y|<\varepsilon \Lambda_{M_{p}}(0, \delta)$, then $\gamma$ can be chosen so that $|\gamma|<\Lambda_{M_{p}}(0, \delta)$ and so that $\eta(1)=y$. This establishes that $R(\varepsilon \delta) \subset B(\delta)$, and completes the proof of Lemma 3.10 .

In $C^{2}$, we have the following lemma, which we will use in $\S 7$ for estimating plurisubharmonic functions in the case when $M$ bounds a pseudoconvex domain. 
Lemma 3.16. Suppose $M$ bounds a pseudoconvex domain in $\mathbb{C}^{2}$. There are positive constants $\delta_{0}$ and $C_{0}$ such that for each $p \in M$ there is a polynomial $\tilde{h}^{p}: C \rightarrow R$ of degree $m_{0}+2$ with no pure terms such that

(a) $\Delta_{w}\left\{\tilde{h}^{p}(w)\right\} \geq 0$ for $w \in C,|w| \leq \delta_{0}$.

(b) For $2 \leq m \leq m_{0}$ and $\alpha, \beta>0$ with $\alpha+\beta=m$

$$
\left|\frac{\partial^{m} \tilde{h}^{p}(0)}{\partial w^{\alpha} \partial \bar{w}^{\beta}}-\frac{\partial^{m} h^{p}(0,0)}{\partial w^{\alpha} \partial \bar{w}^{\beta}}\right| \delta^{m} \leq C_{0} \delta \Lambda_{m}^{h^{p}}(\delta) .
$$

(c) $\left|\Lambda_{m_{0}}^{h^{p}}(\delta)-\Lambda_{m_{0}}^{h^{p}}(\delta)\right| \leq C_{0} \delta \Lambda_{m_{0}}^{h^{p}}(\delta)$.

Proof. We are in the case $n=2$; so there are only two vector fields $L_{1}$ and $L_{2}$ (with $L_{2}=\bar{L}_{1}$ ) which span the complexified holomorphic tangent bundle. We have

$$
\lambda_{1,2} T=\left[L_{2}, L_{1}\right] \bmod \left(L_{1}, L_{2}\right),
$$

where $T=\partial / \partial y$. The pseudoconvexity of $M$ implies $i \lambda_{1,2}^{p}(y, w) \geq 0$ for $(y, w)$ near $(0,0)$. In view of $(3.5)$, we have

$$
i \lambda_{1,2}^{p}(0, w)=2 \frac{\Delta_{w} h^{p}}{\left(1+\left(h_{h}^{p}\right)^{2}\right)}+g_{1},
$$

where $g_{1} \in W_{1}\left(W_{j}\right.$ is the ideal defined in the proof of Lemma 3.2). Let $\tilde{\lambda}_{1,2}^{p}(w)$ be the Taylor polynomial of $\lambda_{1,2}^{p}(0, w)$ of degree $m_{0}$ in powers of $w$ and $\bar{w}$. Let $\widetilde{H}^{p}$ be the polynomial of degree $m_{0}+2$ in $w$ and $\bar{w}$ with

$$
\Delta_{w} \widetilde{H}^{p}=i \tilde{\hat{\lambda}}_{1,2}^{p}(w)
$$

obtained by integrating each term of $\tilde{\lambda}_{1,2}^{p}$ once with respect to $w$ and once with respect to $\bar{w}$. Since $i \lambda_{1,2}^{p}(0, w) \geq 0$, clearly

$$
\Delta_{w} \widetilde{H}^{p} \geq-C|w|^{m_{0}+1}
$$

for some uniform constant $C>0$.

Let

$$
\tilde{h}^{p}(w)=\widetilde{H}^{p}(w)+\frac{C}{\left(\frac{1}{2} m_{0}+1\right)^{2}}|w|^{m_{0}+2} .
$$

The pseudoconvexity of $M$ implies that $m_{0}$ is even and so $\tilde{h}^{p}$ is a smooth polynomial of degree $m_{0}+2$. The constants are arranged so that $\Delta_{w} \tilde{h}^{p}(w) \geq 0$ provided $|w|$ is less than some uniform $\delta_{0}>0$. From the definition of $\tilde{h}^{p}$ and $\widetilde{H}^{p}$ and (3.17) we have

$$
\Delta_{w} \tilde{h}^{p}(w)=2 \frac{\Delta_{w} h^{p}(0, w)}{1+\left(h_{y}^{p}\right)^{2}}+g_{1}+C|w|^{m_{0}}+O\left(|w|^{m_{0}+1}\right)
$$

with $g_{1} \in W^{1}$. Differentiating this equation, we easily establish

$$
\frac{\partial^{j} \tilde{h}^{p}(0)}{\partial w^{\alpha} \partial \bar{w}^{\beta}}=\frac{\partial^{j} h^{p}(0,0)}{\partial w^{\alpha} \partial \bar{w}^{\beta}}+g_{j-1}(0,0) \quad \text { for } 2 \leq \alpha+\beta=j \leq m_{0},
$$


where $g_{j-1} \in W^{j-1}$. Parts (b) and (c) now follow easily from the definition of $\Lambda_{m_{0}}^{h^{p}}(\delta)$. This completes the proof of Lemma 3.16.

Now note that the biholomorphisms $H_{p}$ constructed at the beginning of this section map the set $U^{-}$to the set $\left\{\left(x+h^{p}(y, w)+i y, w\right) ; x \geq 0\right\}$ and $U^{+}$to the set $\left\{\left(x+h^{p}(y, w)+i y, w\right) ; x \leq 0\right\}$. In addition, for $q \in V_{\mu_{0}}^{-}\left(p, \varepsilon_{0} \delta\right)$, note that $H_{p}(q)$ is a point of the form $\left(h^{p}(y, w)+i y, w\right)+(x, 0)$ for some $0<$ $x<\mu_{0} \Lambda_{m_{0}}^{h^{p}}(0, \delta)$, and for some $y \in \mathbb{R}$ and $w \in \mathbb{C}^{n-1}$ with $|y|<\varepsilon_{0} \Lambda_{m_{0}}^{h^{p}}(0, \delta)$ and $|w|<\varepsilon_{0} \delta$. Therefore in view of Lemmas 3.10 and 3.2, Theorem 2.5 (and hence Theorem 2.4) is reduced to

Theorem 3.18. (a) There are positive constants $\mu_{0}, \delta_{0}, \varepsilon_{0}$, and $C$ so that for any $0 \leq \delta \leq \delta_{0}$ there is choice $\eta= \pm 1$ so that for all $w \in C^{n-1}$ and $x, y \in R$ with $|w|<\varepsilon_{0} \delta,|y|<\varepsilon_{0} \Lambda_{m_{0}}^{h^{p}}(0, \delta)$, and $0<\eta x<\mu_{0} \Lambda_{m_{0}}^{h^{p}}(0, \delta)$ there is an analytic disc $A$ so that the restriction of $A$ to $S^{1}$ has image in

$$
\left\{\left(x+h^{p}(y, w)+i y, w\right) ; \eta x \leq 0,|w|<C \delta,|y|<\Lambda_{m_{0}}^{h^{p}}(0, \delta)\right\}
$$

and

$$
A(\zeta=0)=\left(h^{p}(y, w)+i y, w\right)+(x, 0) .
$$

(b) If $M$ is the boundary of a pseudconvex domain, there are constants $\delta_{0}$, $C_{1}$, and $C_{2}$ so that if $0 \leq \delta \leq \delta_{0}$ and $0<x<C_{1} \Lambda_{m_{0}}^{h^{p}}(0, \delta)$, there is an analytic disc $A$ such that the restriction of $A$ to $S^{1}$ has image in

$$
\left\{\left(x+h^{p}(y, w)+i y, w\right) \in M_{p} ; x \leq 0,|w|<C \delta,|y|<\Lambda_{m_{0}}^{h^{p}}(0, \delta)\right\}
$$

and

$$
A(\zeta=0)=(x+i 0,0) .
$$

Furthermore, the constants $\varepsilon_{0}, \mu_{0}, \delta_{0}, C_{1}$, and $C_{2}$ depend only on the $C^{m_{0}+2}$ norm of $\left\{h^{p}, p \in K \subset \subset M\right\}$.

Note that part (b) of Theorem 2.5 follows by applying part (b) of Theorem 3.18 to each point $q \in B_{M}(p, \delta)$ and with $\delta$ in Theorem 3.18 replaced by $\delta_{q p}$ ( $\delta_{q p}$ is defined in Definition 2.3).

\section{THE MODEL CASE}

As we shall see, a rescaling argument will allow us to reduce the proof of Theorem 3.18 to the case where $\delta=1$ and $\Lambda_{m_{0}}^{h^{p}}(0, \delta=1)=1$. In addition, we shall see that the dominant term in $h^{p}(y, w)$ is the Taylor polynomial (expanded about 0 ) of $h^{p}$ in the $w$ and $\bar{w}$ variables of order $m_{0}$. Thus we shall first consider the model case where $h^{p}$ is a polynomial of degree $m_{0}$ in $w$ and $\bar{w}$ with $\Lambda_{m_{0}}^{h^{p}}(0, \delta=1)=1$. 
For a function $h: R \times C^{n-1} \rightarrow R$, define

$$
\Lambda_{m_{0}}^{h}=\Lambda_{m_{0}}^{h}(0, \delta=1) .
$$

We also let $A^{0,1 / 2}\left(S^{1}, C^{n-1}\right)$ denote the space of all $C^{n-1}$ valued Hölder continuous functions (with exponent $\frac{1}{2}$ ) defined on $S^{1}$ whose negative Fourier coefficients vanish. Thus elements of $A^{0,1 / 2}\left(S^{1}, C^{n-1}\right)$ can be identified with certain analytic disc in $C^{n-1}$. We will denote the Hölder norm in this space by $\|\cdot\|$.

We let

$$
S=\left\{\begin{array}{l}
P: C^{n-1} \rightarrow R ; P \text { is a polynomial of degree } m_{0} \\
\text { with no pure terms and with } \Lambda_{m_{0}}^{P}=1 .
\end{array}\right.
$$

The key lemma is the following.

Lemma 4.1. (a) Given a positive integer $m_{0}$, there exists $\mu_{0}>0$ so that for each polynomial $P \in S$ there exists $W \in A^{0,1 / 2}\left(S^{1}, C^{n-1}\right)$ with $\|W\|<1$, and $W(0)=0$ so that

$$
\left|\frac{1}{2 \pi} \int_{0}^{2 \pi} P\left(W\left(e^{i \varphi}\right)\right) d \varphi\right| \geq \mu_{0} .
$$

(b) Given a positive integer $m_{0}$ there exists $\mu_{0}>0$ so that for each subharmonic polynomial $P: \mathbb{C} \rightarrow \mathbb{R}, P \in S$, we have

$$
\frac{1}{2 \pi} \int_{0}^{2 \pi} P\left(e^{i \varphi}\right) d \varphi \geq \mu_{0} .
$$

Remark. Part (b) states that if $P$ is subharmonic, then the conclusion of (a) holds with $W(\zeta)=\zeta$. This will be useful in $\S 6$.

Proof. First, we reduce this lemma to the simpler case when $P$ is a polynomial defined on $C^{1}$.

Sublemma. It suffices to show Lemma 4.1 for the case $n=2$.

Proof. Since $P$ is defined on $C^{n-1}, n \geq 2$, the idea is to examine $P$ along one-dimensional complex lines which pass through the origin. For $v \in C^{n-1}$ with $|v|=1$, we let $P_{n}: C \rightarrow R$ be defined by $P_{v}(w)=P(v w)$ for $w \in C$. We shall assume Lemma 4.1 holds for all the $P_{v} / \Lambda_{m_{0}}^{P_{\prime \prime}} \in S$. Since the constant $\mu_{0}$ in Lemma 4.1 is independent of the polynomial $P \in S$ (for $n=2$ ), it suffices to show that

$$
\sup _{|\eta|=1}\left\{\Lambda_{m_{0}}^{P_{\prime \prime}}\right\} \geq C_{1} \Lambda_{m_{0}}^{p}=C_{1}
$$

holds for any polynomial $P \in S$, where $C_{1}>0$ and is independent of $P$. Our sublemma now follows from the facts that $S$ is compact and the map $v \rightarrow \Lambda_{m_{0}}^{C_{*}}$ for any fixed $\mathscr{Q} \in S$ is a continuous real valued map which is positive for some $v$ with $\|v\|=1$. 
Thus we shall assume our given polynomial $P$ is defined on $C$.

Let $S$ denote the set of polynomials $P(z)=\sum p_{\alpha \beta} z^{\alpha} \bar{z}^{\beta}$ of degree $m_{0}$, with no pure terms and with $\sum\left|p_{\alpha \beta}\right|=1$. Note again that this set is compact. If Lemma 4.1(a) were false, then for each positive integer $n$ we could find a polynomial $P_{n} \in S$ so that

$$
\left|\frac{1}{2 \pi} \int_{0}^{2 \pi} P_{n}\left(W\left(e^{i \varphi}\right)\right) d \varphi\right|<\frac{1}{n}
$$

for every $W \in A^{0,1 / 2}\left(S^{1}, C^{n-1}\right)$ with $\|W\|<1$ and $W(0)=0$. By selecting a convergent subsequence, we conclude that there is a polynomial $P_{0} \in S$ so that

$$
\int_{0}^{2 \pi} P_{0}\left(W\left(e^{i \varphi}\right)\right) d \varphi=0
$$

for every $W \in A^{0,1 / 2}\left(S^{1}, C^{n-1}\right)$ with $\|W\|<1$ and $W(0)=0$. However, we have

Sublemma. Let $P$ be the real analytic function on the closed unit disc $D$ in the complex plane. Suppose

$$
\frac{1}{2 \pi} \int_{0}^{2 \pi} P\left(W\left(e^{i \varphi}\right)\right) d \varphi=0
$$

for every $W \in A^{0,1 / 2}\left(S^{1}, C^{n-1}\right)$ with $\|W\|<1$ and $W(0)=0$. Then $P$ is harmonic.

Assuming this result for the moment, we conclude that $P_{0} \in S$ is harmonic. However, this is a contradiction since $P_{0}$ is not identically zero and has no pure $z$ or $\bar{z}$ terms. Therefore, it suffices to establish the above sublemma.

In fact, the sublemma is true under the weaker hypothesis that $P$ is merely continuous (see Globevnik and Rudin [GR]). For completeness, we give a different proof which works in the real analytic case.

Consider the analytic disc

$$
W_{\lambda, a}(\zeta)=\frac{a \zeta}{1-\lambda \zeta}, \quad a \in C, \quad 0 \leq \lambda<1 .
$$

Clearly, $W_{\lambda, a} \in A^{0,1 / 2}\left(S^{1}, C\right)$ and $\left\|W_{\lambda, a}\right\| \leq C a(1-\lambda)^{-3 / 2}$. Following Boggess, Pitts, and Polking [BPP], we can use Residue Calculus to show that if $m, n \geq 1$, then

$$
\frac{1}{2 \pi} \int_{0}^{2 \pi}\left[W_{\lambda, a}\left(e^{i \varphi}\right)\right]^{m}\left[\bar{W}_{\lambda, a}\left(e^{i \varphi}\right)\right]^{n} d \varphi=a^{m} \bar{a}^{n}\left(A_{m n}\left(1-\lambda^{2}\right)^{-(m+n)+1} E_{m n}(\lambda)\right),
$$

where $\left|E_{m n}(\lambda)\right| \leq\left(1-\lambda^{2}\right)^{-(m+n)+2}$ and the $A_{m n}>0$ are combinatorial constants. If $m$ or $n$ is zero, then

$$
\frac{1}{2 \pi} \int_{0}^{2 \pi}\left[W_{i, a}\left(e^{i \varphi}\right)\right]^{m}\left[\bar{W}_{i \ldots a}\left(e^{i \varphi}\right)\right]^{n} d \varphi=0
$$


because $W_{\lambda, a}(\zeta=0)=0$. Thus if $P$ is real analytic, we can write

$$
P(z)=\sum p_{m n} z^{m} \bar{z}^{n}
$$

and this infinite series converges for $|z|$ small enough. So if

$$
\sup _{|\zeta| \leq 1}\left|W_{\lambda, a}(\zeta)\right| \leq C a(1-\lambda)^{-1}
$$

is sufficiently small, we have

$$
\begin{aligned}
0 & =\frac{1}{2 \pi} \int_{0}^{2 \pi} P\left(W_{\lambda, a}\left(e^{i \varphi}\right)\right) d \varphi \\
& =\sum_{m, n \geq 1} p_{m n} a^{m} \bar{a}^{n}\left[A_{m n}\left(1-\lambda^{2}\right)^{-(m+n)+1}+E_{m n}(\lambda)\right] .
\end{aligned}
$$

Let $k=\inf \left\{s\right.$; there exists $p_{m n} \neq 0$ with $\left.m+n=s\right\}$. If $P$ is not harmonic then $0<k<\infty$ and

$$
0=\left(\sum_{\substack{m+n=k \\ m, n \geq 1}} p_{m n} A_{m n} a^{m} \bar{a}^{n}\right)\left(1-\lambda^{2}\right)^{-k+1}+\left(1-\lambda^{2}\right) O\left(|a|^{k}\left(1-\lambda^{2}\right)^{-k+1}\right)
$$

provided $|a|\left(1-\lambda^{2}\right)^{-1}$ is small. By first choosing $\lambda$ close to 1 and then $|a|$ small, we see that this implies that $p_{m n}=0$ if $m+n=k, m, n \geq 1$, which contradicts the choice of $k$. Therefore, $P$ is harmonic.

For the proof the part (b), we note that $\int_{0}^{2 \pi} P\left(e^{i \varphi}\right) d \varphi>0$ for each subharmonic $P \in S$. Part (b) now follows from the compactness of $S$.

\section{THE General CASE}

For $k \geq 0$ we let $C^{k}\left(\mathbb{R} \times \mathbb{C}^{n-1}\right)$ be the space of complex valued functions on $\mathbb{R} \times \mathbb{C}^{n-1}$ whose $k$ th order derivatives are continuous. A $C^{k}$-neighborhood of a function $h \in C^{k}\left(\mathbb{R} \times \mathbb{C}^{n-1}\right)$ will refer to a neighborhood of $h$ in $C^{k}\left(\mathbb{R} \times \mathbb{C}^{n-1}\right)$ with the usual $C^{k}$ topology, of uniform convergence of $k$ th order derivatives on compact subsets of $\mathbb{R} \times \mathbb{C}^{n-1}$.

The key lemma is the following.

Lemma 5.1. (a) There are constants $\varepsilon_{0}, \mu_{0}>0$ and a neighborhood $U$ of the origin in $C^{0}\left(\mathbb{R} \times \mathbb{C}^{n-1}\right)$ so that for any $P \in S$ ( $S$ defined in $\left.\S 4\right)$ there is a family of analytic discs $A(y, w): \bar{D} \rightarrow \mathbb{C} \times \mathbb{C}^{n-1}$ for $|y|,|w|<\varepsilon_{0}$ of the form $A(y, w)(\zeta)=(G(y, w)(\zeta), W(w)(\zeta)) \in \mathbb{C} \times \mathbb{C}^{n-1}$ so that for any $h \in C^{0}\left(\mathbb{R} \times \mathbb{C}^{n-1}\right)$ with $h-P \in U$

(i) $A(y, w)(\zeta=0)=\left(x^{*}+i 0,0\right)+(h(y, w)+i y, w)$ with $\left|x^{*}\right|>\mu_{0}$,

(ii) $\operatorname{sgn}\left(x^{*}\right) \operatorname{Re} G(y, w)(\zeta)<h(\operatorname{Im} G(y, w)(\zeta), W(w)(\zeta))$ for $|\zeta|=1$.

(b) There are constants $\varepsilon_{0}, \mu_{0}>0$ so that for any $P \in S$ with $P: \mathbb{C} \rightarrow \mathbb{R}$ subharmonic, the conclusion of part (a) holds with $W(w)(\zeta)=\zeta+w$ and $x^{*}>$ $\mu_{0}$. 
Proof. For part (a), we note first that we can apply Lemma 4.1(a) to obtain $\mu_{0}>0$ so that for any $P \in S$ there is an analytic disc $\widetilde{W}: \widetilde{D} \rightarrow \mathbb{C}^{n-1}$ with $\widetilde{W}(\zeta=0)=0$ and $|I| \geq \mu_{0}$, where

$$
I=\frac{1}{2 \pi} \int_{0}^{2 \pi} P\left(\widetilde{W}\left(e^{i \varphi}\right)\right) d \varphi .
$$

Let $\widetilde{G}: D \rightarrow \mathbb{C}$ be an analytic function with

$$
\operatorname{Re} \widetilde{G}\left(e^{i \varphi}\right)=P\left(\widetilde{W}\left(e^{i \varphi}\right)\right)-I, \quad \widetilde{G}(0)=0 .
$$

Such a $\widetilde{G}$ exists since the mean value of the right side of the first equation above (over $0 \leq \varphi \leq 2 \pi$ ) is zero. Let

$$
A(\zeta)=(G(y, w)(\zeta), W(w)(\zeta)),
$$

where $G(y, w)(\zeta)=\widetilde{G}(\zeta)+\frac{1}{3} I+i y$ and $W(w)(\zeta)=\widetilde{W}(\zeta)+w$. Since $\widetilde{G}(\zeta=$ $0)=0$ and $\widetilde{W}(\zeta=0)=0$, we have $A(\zeta=0)=\left(\frac{1}{3} I+i y, w\right)=\left(\frac{1}{3} I-\right.$ $h(y, w), 0)+(h(y, w)+i y, w)$. We can restrict $\varepsilon_{0}>0$ suitably small so that if $|y|,|w|<\varepsilon_{0}$, then $|h(y, w)|<\mu_{0} / 6$. Together with the estimate $\left|\frac{1}{3} I\right| \geq \mu_{0} / 3$, we see that (i) holds with $\mu_{0}$ replaced by $\mu_{0} / 6$ and $x^{*}=\frac{1}{3} I-h(y, w)$.

To show (ii), we first note

$$
\|\widetilde{G}\| \leq C \Lambda_{m_{0}}^{P}(0,\|W\|) \leq C,
$$

where $C$ is a positive constant independent of $P \in S$. This is easily shown by noting $(5.2)$ and $\operatorname{Im} \widetilde{G}=T(\operatorname{Re} \widetilde{G}) \quad(T=$ Hilbert transform $)$ and by recalling that $T$ is continuous with respect to $C^{0,1 / 2}$ norm $(\|\cdot\|)$.

Now suppose $I \geq \mu_{0}>0$. For $|\zeta|=1$, we have

$$
\begin{aligned}
\operatorname{Re} G(y, w)(\zeta) & =\operatorname{Re} \widetilde{G}(\zeta)+\frac{1}{3} I \\
& =P(\widetilde{W}(\zeta))-\frac{2}{3} I \quad(\text { by }(5.2)) \\
& \leq h(\operatorname{Im} \widetilde{G}(\zeta), \widetilde{W}(\zeta))-\frac{1}{3} I,
\end{aligned}
$$

where (in view of $(5.3)$ and $\|\widetilde{W}\| \leq 1$ ) the last inequality holds for any $h$ with $h-P \in U$ provided $U$ is a sufficiently small neighborhood of the origin in $C^{0}\left(\mathbb{R} \times \mathbb{C}^{n-1}\right)$. Since $S$ is compact, this neighborhood can be taken to be independent of $P \in S$. Thus if $|y|,|w|<\varepsilon_{0}$ and $\varepsilon_{0}$ is small enough,

$$
\operatorname{Re} G(y, w)(\zeta) \leq h(\operatorname{Im} G(y, w)(\zeta), \widetilde{W}(\zeta)+w)
$$

which proves (ii) for the case $I \geq \mu_{0}$. The case $I \leq-\mu_{0}$ is handled in a similar manner.

The proof of part (b) follows in the same way as part (a) except part (b) of Lemma 4.1 is used instead of Lemma 4.1(a). This completes the proof.

Now we complete the proof of Theorem 3.18 (and hence Theorem 2.4). For $\delta>0$ define $H_{\delta}^{p}: \mathbb{C} \times \mathbb{C}^{n-1} \rightarrow \mathbb{C} \times \mathbb{C}^{n-1}$ by $H_{\delta}^{p}(z, w)=\left(\Lambda_{m_{0}}^{h^{p}}(0, \delta) z, \delta w\right)$, 
and define

$$
h_{\delta}^{p}=\frac{h^{p} \circ H_{\delta}^{p}}{\Lambda_{m_{0}}^{h^{p}}(0, \delta)}
$$

and

$$
P_{\delta}^{p}(w)=\sum_{2 \leq|\alpha|+|\beta| \leq m_{0}} \frac{\partial^{|\alpha|+|\beta|} h_{\delta}^{p}(0,0)}{\partial w^{\alpha} \partial \bar{w}^{\beta}} w^{\alpha} \bar{w}^{\beta} .
$$

We note $\Lambda_{m_{0}}^{h_{\delta}^{p}}=\Lambda_{m_{0}}^{P_{\delta}^{p}}=1$ and

$$
\left|h_{\delta}^{p}(y, w)-P_{\delta}^{p}(w)\right| \leq C\left(|y||w|+|w|^{m_{0}+1}\right) \delta,
$$

where the last inequality can be easily verified by Taylor expanding $h_{\delta}^{p}$ about 0 in $w$ and $\bar{w}$ and by using Lemma 3.9. Thus given any $C^{0}$-neighborhood $U$ of the origin, clearly $\delta_{0}$ can be chosen small enough so that $h_{\delta}^{p}-P_{\delta}^{p} \in U$ for $0<\delta \leq \delta_{0}$.

Note that $H_{\delta}^{p}$ takes the set $\left\{x=h_{\delta}^{p}(y, w)\right\}$ to $M^{p}=\left\{x=h^{p}(y, w)\right\}$ and that $H_{\delta}^{p}$ takes a set of the form $\left\{x=h_{\delta}^{p}(y, w) ;|y|,|w|<C\right\}$ onto a set which is comparable to $B_{M_{p}}(0, \delta)$ (in view of Lemma 3.10). Thus it suffices to show Theorem 3.18 holds with $h^{p}$ replaced by $h_{\delta}^{p}\left(0<\delta \leq \delta_{0}\right)$ and with $\Lambda_{m_{0}}^{h^{p}}(0, \delta)$ replaced by $\Lambda_{m_{0}}^{h_{\dot{\delta}}^{p}}$.

Now for any fixed $0<\delta \leq \delta_{0}$ we can use Lemma 5.1 to obtain an analytic disc $A(y, w)(\zeta)=(G(y, w)(\zeta), W(w)(\zeta))$ satisfying (i) and (ii) for $|y|,|w|<$ $\varepsilon_{0}$. Now $\varepsilon_{0}$ and $\mu_{0}$ in Lemma 5.1 are independent of $P \in S$ and therefore are independent of $0<\delta \leq \delta_{0}$.

Suppose $x^{*} \geq \mu_{0}\left(x^{*}\right.$ as in Lemma 5.1). For $0 \leq s \leq x^{*}$ we let

$$
A(y, w, s)(\zeta)=(G(y, w)(\zeta)-s, W(w)(\zeta)) .
$$

Note that (ii) (in Lemma 5.1(a)) holds for $A(y, w, s)(\zeta)$. Also

$$
A(y, w, s)(\zeta=0)=\left(x^{*}-s+i 0,0\right)+(h(y, w)+i y, w) .
$$

Thus by choosing $s$ appropriately, we see that any point of the form $(x+$ $\left.h_{\delta}^{p}(y, w)+i y, w\right)$ with $0 \leq x \leq \mu_{0}$ and $|y|,|w|<\varepsilon_{0}$ is the center of an analytic disc with boundary in the set

$$
\{(x+i y, w) ; x \leq h(y, w) \text { and }|y|,|w|<C\},
$$

where $C$ is a constant independent of $\delta$. This proves Theorem 3.18 for the case $x^{*} \geq \varepsilon_{1}$ (i.e., take $\eta=+1$ ). The case when $x^{*} \leq-\mu_{0}$ is handled in a similar way (leading to the choice $\eta=-1$ ).

Part (b) of Theorem 3.18 follows from part (a) of Theorem 3.18 because the pseudoconvexity of $M$ implies that the hull of $B_{M_{p}}(0, \delta)$ must always lie on one side of $M_{p}$ regardless of $\delta$. This means we only have the case $\eta=+1$.

\section{ESTIMATING PLURISUBHARMONIC FUNCTIONS}

By the same change of variables argument with $H_{p}$ as in $\S 3$ which was used to reduce Theorem 2.5 to Theorem 3.18 , we can reduce Theorem 2.6 to 
Lemma 6.1. There are constants $\delta_{0}, C, A>0$ with the following property. For any $p \in M$, let $u$ be a plurisubharmonic function on the pseudoconvex set $\left\{(x+i y, w) \in \mathbb{C}^{n} ; x>h^{p}(y, w)\right\}$ and continuous on the closure of this set. For any $0<\delta \leq \delta_{0}$ and $x=\Lambda_{M_{p}}(0, \delta)$ we have

$$
u(x+i 0,0) \leq \frac{A}{\left|B_{p}\right|} \int_{\zeta \in B_{p}}|u(\zeta)| d \sigma(\zeta)
$$

where $B_{p}=B_{M_{p}}(0, \delta)$.

By a slicing argument we need only prove this for $n=2$. As in $\S 5$, we shall rescale by using the map $H_{\delta}^{p}$ which will allow us to reduce the proof to the case $\delta=1$. Therefore the key lemma is

Lemma 6.2. There is a neighborhood $U$ of the origin in $C^{1}(\mathbb{R} \times \mathbb{C})$ and constants $x^{*}, C>0$ and a compact set $K \subset \mathbb{R} \times \mathbb{C}$ so that for any $P \in S$ and any $h \in C^{1}(\mathbb{R} \times \mathbb{C})$ with $h-P \in U$ we have

$$
u\left(x^{*}+i 0,0\right) \leq \int_{(y, w) \in K}|u(h(y, w)+i y, w)| d y d \lambda(w)
$$

for each $u$ which is plurisubharmonic on $\{x>h(y, w)\}$ and continuous on $\{x \geq h(y, w)\}$.

Here $d \lambda(w)$ is Lebesgue measure on $\mathbb{C}$.

Proof. Since $\{P \in S ; P$ is subharmonic $\}$ is compact, it suffices to show that a neighborhood $U$ as above exists for each fixed $P$.

Using Lemma 5.1(b) with $y, w=0$, we can find an analytic disc $A(\zeta)=$ $(G(\zeta), \zeta)$, a map $A: \bar{D} \rightarrow \mathbb{C} \times \mathbb{C}$, and a $C^{0}$-neighborhood $\widetilde{U}$ of $P$ so that for any $h \in \widetilde{U}$

(i) $A(\zeta=0)=\left(x^{*}+i 0,0\right)$ with $x^{*}>0$,

(ii) $\operatorname{Re} G(\zeta)<h(\operatorname{Im} G(\zeta), \zeta)$ for $|\zeta|=1$.

Now (i) and (ii) remain true for small real perturbations of $G$ of $h$. So we shall perturb $G$ so that for each $h$ in some $C^{1}$-neighborhood of $P$

(iii) the curve $\{\zeta \in D ; A(\zeta) \in M\}$ is a closed simple curve, where $M=$ $\{x=h(y, w)\}$ and $A(\zeta)=(G(\zeta), \zeta)$.

Sublemma. There exists an analytic disc $G: \bar{D} \rightarrow \mathbb{C}$ satisfying (i), (ii) and (iii). Proof of Sublemma. It suffices to find such a $G$ satisfying (i), (ii), and (iii) for $h=P$; for then (i), (ii), and (iii) will certainly hold for all $h$ in some small $C^{1}$ neighborhood of $P$.

For $G: \bar{D} \rightarrow D$ analytic let

$$
\varphi_{G}(\zeta)=\operatorname{Re} G(\zeta)-P(\zeta), \quad \zeta \in \bar{D}
$$

With $G$ defined as above satisfying (i) and (ii), choose $\zeta_{0} \in D$ so that $\varphi_{G}\left(\zeta_{0}\right)=$ 0 . The point $\zeta_{0} \in D$ exists because $\{x=P(w)\}$ separates $\mathbb{C}^{2}$ and because $\varphi_{G}(\zeta=0)=x^{*}>0$ and $\varphi_{G}(\zeta)<0$ for $|\zeta|=1$. 
We may assume $\partial \varphi_{G}\left(\zeta_{0}\right) / \partial x \neq 0$, where $\zeta=x+i y$. For otherwise we set $G^{\varepsilon}(\zeta)=G(\zeta)+\varepsilon\left(\zeta-\zeta_{0}\right) \zeta$, with $\varepsilon \in \mathbb{C}$ chosen small so that $\partial \varphi_{G^{\varepsilon}}\left(\zeta_{0}\right) / \partial x \neq 0$. Note that (i) and (ii) still hold for $G^{\varepsilon}$ provided $|\varepsilon|$ is sufficiently small.

Since $\partial \varphi_{G}\left(\zeta_{0}\right) / \partial x \neq 0$, clearly $\varphi_{G}$ maps a small neighborhood of $\zeta_{0}$ to a small neighborhood of $\varphi_{G}\left(\zeta_{0}\right)=0$ in $\mathbb{R}$. Sard's theorem implies that the image under $\varphi_{G}$ of the set of critical points of $\varphi_{G}$ is a set of measure zero in $\mathbb{R}$. Therefore we can choose $\alpha \in$ Image $\varphi_{G}$ arbitrarily small so that

$$
\varphi_{G}^{-1}(\alpha) \cap\left\{\text { set of critical points of } \varphi_{G}\right\}=\varphi .
$$

The curve $\varphi_{G}^{-1}(\alpha)$ is thus a closed simple curve in $D$. In addition, the curve $\varphi_{G}^{-1}(\alpha)$ is the set $\left\{\zeta \in D ; A_{\alpha}(\zeta) \in M_{0}\right\}$, where $M_{0}=\{x=P(w)\}$ and where $A_{\alpha}(\zeta)=(G(\zeta)-\alpha, \zeta)$. So, if $\alpha$ is sufficiently small, then $A^{\alpha}$ satisfies (i), (ii), and (iii), as desired. This completes the proof of the sublemma.

Now clearly, small complex perturbations of $G$ also satisfy (ii) and (iii). Therefore we shall choose $\varepsilon>0$ small so that if $|x+i y|<\varepsilon$, then (ii) and (iii) hold for the analytic disc $\zeta \mapsto G(\zeta)+x+i y$.

If $u$ is plurisubharmonic on $V^{+}=\{x>h(y, w)\}$ then we can subaverage $u$ and obtain

$$
u\left(x^{*}+i 0,0\right) \leq \frac{1}{\pi \varepsilon^{2}} \int_{(x+i y) \in D_{\varepsilon}} u\left(x^{*}+x+i y, 0\right) d x d y,
$$

where $D_{\varepsilon}=\{(x+i y) \in \mathbb{C} ;|x+i y|<\varepsilon\}$. Here we shall assume that $\varepsilon<x^{*} / 2$ so that we ensure that $u(z, 0)$ is subharmonic for $z \in\left\{x^{*}+D_{\varepsilon}\right\}$. Note that the constant $C=1 / \pi \varepsilon^{2}$ depends only on the $C^{1}$-neighborhood of $P$ but is independent of $h$ in this neighborhood and the plurisubharmonic $u$.

Let $A(x, y)(\zeta)=(G(\zeta)+x+i y, \zeta)$ and let

$$
C_{x y}=\{\zeta \in D, A(x, y)(\zeta) \in M\} \quad(\text { where } M=\{x=h(y, w)\}) .
$$

For $(x+i y) \in D_{\varepsilon}, C_{x y}$, is a simple close curve in $D$ (from (iii)) which separates $D$ into two components $\left\{\zeta \in D ; A(x, y)(\zeta) \in V^{+}\right\}$and $\{\zeta \in D ; A(x, y)(\zeta) \notin$ $\left.V^{+}\right\}$; both with boundary equal to $C_{x y}$. Since $u \circ A(x, y)$ is subharmonic on the former and since $A(x, y)(\zeta=0)=\left(x^{*}+x+i y, 0\right)$ we have from $(6.3)$

$$
u\left(x^{*}+i 0,0\right) \leq C \int_{\zeta \in C_{x y}} \int_{(x, y) \in D_{z}}|u(G(\zeta)+x+i y, \zeta)| d x d y d \sigma_{x y}(\zeta),
$$

where $d \sigma_{x y}$ denotes surface measure (i.e., arc length) on $C_{x y}$ and where $C$ is a constant which is independent of $h \in U+P$ and $u$.

Now the map

$$
(x, y, \zeta) \mapsto(G(\zeta)+x+i y, \zeta) \in M
$$

for $\zeta \in C_{x y}$ and $(x+i y) \in D_{\varepsilon}$ is a $C^{1}$ map with nonsingular derivative and so this map is locally invertible and its image contains an open subset of $M$. Furthermore its image is contained in some compact subset $\widetilde{K} \cap M$ of $M$. So

$$
u\left(x^{*}+i 0,0\right) \leq C \int_{M \cap \widetilde{K}}|u| d \sigma,
$$

where $d \sigma$ is surface measure on $M$. 
Finally, the map $(x+i y, w) \rightarrow(x+h(y, w)+i y, w)$ is a diffeomorphism which takes $\{x=0\}$ to $M$. Therefore

$$
u\left(x^{*}+i 0,0\right) \leq C \int_{(y, w) \in K} \mid u(h(y, w)+i y, w) d y d \lambda(w),
$$

where $K$ is a compact subset of $\mathbb{R} \times \mathbb{C}$. The set $K$ and constant $C$ depend only on the $C^{1}$ neighborhood of $P$ as desired. This completes the proof of Lemma 6.2.

Now we complete the proof of Lemma 6.1 (and therefore Theorem 2.6). We let $h^{p}$ and $\tilde{h}^{p}$ be defined as in $\S 3$ (see Lemma 3.16 for the properties of $\tilde{h}^{p}$ ). We define $H_{p}^{\delta}: \mathbb{C}^{2} \rightarrow \mathbb{C}^{2}$

$$
H_{p}^{\delta}(z, w)=\left(\Lambda_{m_{0}}^{\dot{h}_{\dot{\delta}}^{p}}(0, \delta) z, \delta w\right)
$$

and we let

$$
h_{\delta}^{p}=\frac{h^{p} \circ H_{\delta}^{p}}{\Lambda_{m_{0}}^{\hat{h}^{p}}(0, \delta)}, \quad P_{\delta}^{p}=\frac{\tilde{h}^{p} \circ H_{\delta}^{p}}{\Lambda_{m_{0}}^{h^{p}}(0, \delta)} .
$$

As in $\S 5$, we note that $H_{\delta}^{p}$ takes $\left\{x>h_{\delta}^{p}(y, w)\right\}$ to $\left\{x>h^{p}(y, w)\right\}$. We also note

(i) $\Lambda_{m_{0}}^{P_{\delta}^{p}}=1 \quad\left(\right.$ so $\left.P_{\delta}^{p} \in S\right)$;

(ii) $P_{\delta}^{p}$ is subharmonic;

(iii) $P_{\delta}^{p}-h_{\delta}^{p} \rightarrow 0$ as $\delta \rightarrow 0$, where the convergence takes place in $C^{\infty}(\mathbb{R} \times \mathbb{C})$.

Facts (i) and (ii) are immediate from the definitions. Part (iii) is seen by a Taylor expansion of $P_{\delta}^{p}$ and $h_{\delta}^{p}$ and by using Lemma 3.16(b).

Therefore there is a $\delta_{0}>0$ so that $P_{\delta}^{p}-h_{\delta}^{p} \in U$ for $0<\delta \leq \delta_{0}$, where $U$ is the $C^{1}$ neighborhood found in Lemma 6.2. For $u$ a plurisubharmonic function on $\left\{x>h^{p}(y, w)\right\}$ and for $0<\delta \leq \delta_{0}$ define $u_{\delta}^{p}=u \circ H_{\delta}^{p}$ which is plurisubharmonic on $\left\{x>h_{\delta}^{p}(y, w)\right\}$. In view of (i), (ii), and (iii) above, we can apply Lemma 6.2 to $u_{\delta}^{p}$ and $h_{\delta}^{p}$ to conclude

$$
u_{\delta}^{p}\left(x^{*}+i 0,0\right) \leq C \int_{(y, w) \in K} \mid u_{\delta}^{p}\left(h_{\delta}^{p}(y, w)+i y, w\right) d y d \lambda(w) .
$$

Now, we pull back the integral on the right via $\left(H_{\delta}^{p}\right)^{-1}$ to obtain

$$
\begin{aligned}
& u\left(\Lambda_{m_{0}}^{\dot{h}^{p}}(0, \delta) x^{*}+i 0,0\right) \\
& \quad \leq \frac{C}{\delta^{2} \Lambda_{m_{0}}^{h^{\prime \prime}}(0, \delta)} \int_{\substack{|y| \leq C \Lambda_{m_{0}}^{h^{\prime}}(0, \delta) \\
|w| \leq C \delta}} \mid u\left(h^{p}(y, w)+i y, w\right) d y d \lambda(w) .
\end{aligned}
$$

In view of Lemmas 3.16(c) and 3.2, $\Lambda_{m_{0}}^{h^{p}}(0, \delta) \approx \Lambda_{M_{r}}(0, \delta)$ and in view of Lemma 3.10 the ball $B_{M_{n}}(0, \delta)$ is comparable to the graph on $M_{p}$ of the domain of integration on the right side of the above inequality. Finally we note $\left|B_{M_{p}}(0, \delta)\right| \approx \delta^{2} \Lambda_{m_{0}}^{h^{\prime \prime}}(0, \delta)$, and so the proof of Lemma 6.1 is complete. 


\section{BIBLIOGRAPHY}

[BT] M. S. Baouendi and F. Treves, A property of the functions and distributions annihilated by a locally integrable system of complex vector fields, Ann. of Math. (2) 113 (1981), 387-421.

[Bi] E. Bishop, Differential manifolds in complex Euclidean space, Duke Math. 32 (1965), 1-21.

[BG] T. Bloom and I. Graham, A geometric characterization of points of type $m$ on real hypersurfaces, J. Differential Geom. 12 (1977), 171-182.

[BP] A. Bogess and J. T. Pitts, CR extensions near a point of higher type, Duke Math. J. 52 (1985), 67-102.

[BPP] A. Boggess, J. Pitts, and J. Polking, The local hull of holomorphy of semirigid submanifolds of codimension two, Michigan Math. J. 34 (1987), 105-118.

[GR] J. Globevnik and W. Rudin, A characterization of harmonic functions, preprint.

[HT] C. D. Hill and G. Taiani, Families of analytic disc in $C^{n}$ with boundary as prescribed $C R$ submanifold, Ann. Scuola Norm Sup. Pisa 4-5 (1978), 327-380.

[K] J. J. Kohn, Boundary behavior of $\bar{\partial}$ on weakly pseudoconvex manifolds of dimensional two, J. Differential Geom. 6 (1972), 523-542.

[L] H. Lewy, On the character of the solution of an atypical differential equation in three variables and a related problem for regular functions of two complex variables, Ann. of Math. (2) 64 (1956), 514-522.

[N] A. Nagel, Vector fields and nonisotropic metrics, Bejing Lectures in Harmonic Analysis (E. M. Stein, ed.), Ann. of Math. Studies, no. 112, Princeton Univ. Press, Princeton, N. J., 1986.

[NSW1] A. Nagel, E. Stein, and W. Wainger, Boundary behavior of holomorphic functions in domains of finite type, Proc. Nat. Acad. Sci. U.S.A. 78 (1981), 6596-6599.

[NSW2] __ Balls and metrics defined by vector fields I; Basic properties, Acta Math. 155 (1985), 103-147.

Department of Mathematics, Texas A \& M University, College Station, Texas 77843

Institute of Mathematics, Pkin, Warsaw, Poland 00-91

Department of Mathematics, University of Wisconsin, Madison, Wisconsin 53706 